\title{
Función relacional, comunicacional y sinérgica de las Relaciones Públicas en las organizaciones públicas y privadas del Perú
}

Recibido: 06 de agosto de 2014

Aceptado: 24 de febrero de 2015

Publicado: 30 de octubre de 2015
Miguel Valdez Orriaga

vvaldezo@usmp.pe

Universidad de San Martín de Porres (Perú)

Resumen: La presente investigación propone la articulación, desde una concepción de conjunto, de un nuevo modelo teórico basado en el establecimiento de tres funciones de Relaciones Públicas que deben estar presentes en las organizaciones públicas y privadas: la función relacional, la función comunicacional y la función sinérgica. Es un estudio exploratorio, no experimental, descriptivo, comparativo y de modalidad intracultural, que abordó a una muestra de 20 responsables de Relaciones Públicas y afines de organizaciones públicas y privadas del Perú. Entre los principales resultados destacan el alto nivel de gestión de la relación humana, de la administración de la controversia pública y de la subfunción del mensaje unidireccional sobredimensionado en las organizaciones privadas de Lima. En cambio, en las organizaciones públicas de provincias se registró un nivel bajo en esos aspectos.

Palabras claves: Función relacional, función comunicacional, función sinérgica, relación humana, relaciones de poder, administración de la controversia pública.

Abstract: This investigation led proposes the articulation, in a conception as a whole, of a new theoretical model based on the stablishment of three functions of Public Relations that should be present in public and private organizations, that are, the relational function, the communicational function and the synergy function. This is an exploratory study, non-experimental, descriptive, comparative, and from an intracultural mode that took a sample of twenty executive officers of Public Relations and related, from public and private organizations in Peru. Among the main results it was found a high level of management in human relations in private organizations in Lima, but a low level in other cities of the country. These high and low levels were found in the management of the public controversy in organizations of Lima and provinces, respectively. Likewise, 
a high level of the subfunction unidirectional oversized message was found in private organizations in Lima, and rather low in provinces.

Key words: Relational Function, Communicational Function, Synergy Function, Human Relations, Power Relations, Public Controversy Management.

\section{Introducción}

Este estudio ha hecho una revisión de los fundamentos teóricos, epistemológicos y prácticos de las Relaciones Públicas desde las doctrinas norteamericana, europea y latinoamericana que le han dado importante vigencia a esta disciplina. En ese sentido, se propone la articulación (desde una concepción de conjunto) de un nuevo modelo teórico basado en el establecimiento de tres funciones de RR. PP. que pueden ser aplicadas en organizaciones tanto públicas como privadas en el país: la relacional, la comunicacional y la sinérgica, dándose un especial énfasis y autonomía a la primera función, que se traduce en la gestión de la relación humana que le da legitimidad a la acción comunicacional de las organizaciones.

En esta investigación, la función relacional se construye a partir de la propuesta inicial de la Escuela Europea de RR. PP. que le da a la disciplina un enfoque antropológico y sociológico rescatando al ser humano como eje en la dinámica y el comportamiento de las organizaciones que ha de entroncarse en la estrategia de la confianza (Noguero, 2007). También se ha tenido en cuenta a Grunig que rescata hoy el enfoque relacional al proponer indicadores para su medición (confianza, control mutuo, satisfacción y compromiso), aun cuando no profundiza en su fundamentación teórica (ápud Solórzano Castro, 2007).

De igual modo, se ha articulado la teoría relacional propuesta inicialmente por Teobaldo de Souza Andrade a través de la administración de la controversia pública como instrumento metodológico de RR. PP., quien recomienda a los empresarios desarrollar capacidades y habilidades para presentar dicha controversia, discutirla, interpretarla y definirla, vía el diálogo planificado y permanente entre la administración y los grupos ligados directa o indirectamente a las empresas (ápud Solórzano Hernández, 2013: 86). Asimismo, se ha tenido en cuenta la teoría relacional que enriquece el constructo de la administración de la controversia pública conducente a la construcción de relaciones y a la búsqueda de consensos en el sistema organización-públicos.

En la construcción de la función comunicacional, este estudio ha considerado a Grunig y Hunt (2000), quienes presentaron a la comunidad científica el paradigma de los "cuatro modelos" de RR. PP. (agente de prensa, información pública, asimétrico-bidireccional y simétrico-bidireccional) que describen y explican la evolución de la disciplina desde 1850 hasta 1980 desde el punto de vista histórico. Finalmente, se construyó la variable "función sinérgica", la cual hará posible la puesta en marcha de las otras funciones (relacional y comunicacional). Esta función sinérgica (que comprende los roles estratégico y gerencial de un área de RR. PP.), está basada en la teoría de la excelencia planteada por Grunig (2006). 
Esta investigación plantea tres problemas generales: a) ¿qué diferencias existen en la función relacional que utilizan los responsables de RR. PP. de las organizaciones públicas y privadas del Perú?; b) ¿qué diferencias existen en la función comunicacional que utilizan los responsables de RR. PP. de las organizaciones públicas y privadas del Perú?; y c) ¿qué diferencias existen en la función sinérgica que ejercen los responsables de RR. PP. de las organizaciones públicas y privadas del Perú?

El aporte teórico-práctico considera que el ejercicio cabal de las RR. PP., a nivel de las organizaciones públicas y privadas, debe darse con la presencia de las funciones relacional y comunicacional (binomio inseparable) debidamente articuladas por la función sinérgica, a través de un adecuado rol estratégico y gerencial de RR. PP.

\section{Objetivos}

A continuación, se muestra la siguiente relación de objetivos generales y específicos, entrelazados con las tres funciones de RR. PP.:

Figura 1: Objetivos de la investigación.

\begin{tabular}{|c|c|}
\hline \begin{tabular}{|l|} 
OBJETIVOS \\
GENERALES
\end{tabular} & OBJETIVOS ESPECÍFICOS \\
\hline $\begin{array}{l}\text { I. Comparar la } \\
\text { función relacional } \\
\text { que utilizan los } \\
\text { responsables de } \\
\text { RR. PP. de las } \\
\text { organizaciones } \\
\text { públicas y privadas } \\
\text { del Perú. }\end{array}$ & $\begin{array}{l}\text { - Comparar la subfunción gestión de la relación humana promovida por los responsables } \\
\text { de RR. PP. de las organizaciones públicas y privadas del Perú. } \\
\text { - Comparar la subfunción gestión de las relaciones de poder de los responsables de RR. } \\
\text { PP. de las organizaciones públicas y privadas del Perú. } \\
\text { - Comparar la subfunción proceso de administración de la controversia pública utilizada } \\
\text { por los responsables de RR. PP. de las organizaciones públicas y privadas del Perú. }\end{array}$ \\
\hline $\begin{array}{l}\text { II. Comparar } \\
\text { la función } \\
\text { comunicacional de } \\
\text { los responsables } \\
\text { de RR. PP. de las } \\
\text { organizaciones } \\
\text { públicas y privadas } \\
\text { del Perú. }\end{array}$ & $\begin{array}{l}\text { - Comparar la subfunción mensajes de comunicación unidireccional } \\
\text { sobredimensionada que utilizan los responsables de RR. PP. de las organizaciones } \\
\text { públicas y privadas del Perú. } \\
\text { - Comparar la subfunción mensajes de comunicación unidireccional racional que utilizan } \\
\text { los responsables de RR. PP. de las organizaciones públicas y privadas del Perú. } \\
\text { - Comparar la subfunción rol de comunicación bidireccional sobredimensionado que } \\
\text { desempeñan los responsables de RR. PP. de las organizaciones públicas y privadas } \\
\text { del Perú. } \\
\text { - Comparar la subfunción rol de comunicación bidireccional racional que desempeñan } \\
\text { los responsables de RR. PP. de las organizaciones públicas y privadas del Perú. } \\
\text { Comparar la subfunción uso de los medios tradicionales promovido por los } \\
\text { responsables de RR. PP. de las organizaciones públicas y privadas del Perú. } \\
\text { Comparar la subfunción uso de los medios online promovido por los responsables de RR. PP. } \\
\text { de las organizaciones públicas y privadas del Perú. }\end{array}$ \\
\hline
\end{tabular}


III. Comparar la función sinérgica que ejercen los responsables de RR. PP. de las organizaciones públicas y privadas del Perú.
- Comparar la subfunción rol estratégico de RR. PP. de las organizaciones públicas y privadas del Perú.

- Comparar la subfunción rol gerencial de RR. PP. de las organizaciones públicas y privadas del país.

Fuente: Elaboración propia.

\section{Método}

\subsection{Diseño metodológico}

El estudio corresponde a un enfoque cuantitativo y exploratorio no experimental, dado que no se manipulan variables. Asimismo, es descriptiva y comparativa porque se busca comparar variables entre grupos. Posee una modalidad intracultural, ya que se analizará en diferentes regiones del Perú.

Por ser exploratoria, la presente investigación no tiene hipótesis toda vez que se ha tenido que construir instrumentos propios para la medición de las variables correspondientes. Como se sabe, las investigaciones con hipótesis están fundamentadas en sólidas bases teóricas y antecedentes de la investigación, lo cual permite al investigador hacer conjeturas sobre futuros hallazgos.

\subsection{Diseño muestral}

\subsubsection{Población}

La población correspondiente a esta investigación es de 385 relacionistas públicos de organizaciones públicas y privadas, según información del Colegio Profesional de Relacionistas Públicos del Perú.

\subsubsection{Muestra}

La selección de los elementos muestrales se realizó sin ningún procedimiento matemático, es decir cada elemento que formó parte de la muestra fue seleccionado en forma intencionada (criterio del investigador, criterio opinático), respetando así el muestreo no probabilístico o subjetivo, ya que este no somete la selección de los elementos de la muestra a ninguna aleatorización bajo ningún medio tecnificado o mecánico. 
Figura 2: Características de la muestra final.

\begin{tabular}{|c|c|c|c|}
\hline \multicolumn{2}{|c|}{ LIMA } & \multicolumn{2}{|c|}{ PROVINCIAS } \\
\hline PÚBLICAS & PRIVADAS & PÚBLICAS & PRIVADAS \\
\hline $\begin{array}{l}\text { - } \text { Cartera ministerial A. } \\
\text { - } \text { Cartera ministerial B. } \\
\text { - Hobierno local. } \\
\text { - } \begin{array}{l}\text { Organismo público } \\
\text { descentralizado. }\end{array}\end{array}$ & $\begin{array}{l}\text { - } \begin{array}{l}\text { Corporación } \\
\text { latinoamericana de } \\
\text { belleza. }\end{array} \\
\text { - } \begin{array}{l}\text { Constructora } \\
\text { transnacional. }\end{array} \\
\text { - Grupo hospitalario. } \\
\text { - Asociación de } \\
\text { empresas. } \\
\text { - Universidad privada. }\end{array}$ & $\begin{array}{ll}\text { - } & \begin{array}{l}\text { Poder del Estado } \\
\text { (La Libertad). }\end{array} \\
\text { - } & \begin{array}{l}\text { Gobierno local } \\
\text { (Junín) }\end{array} \\
\text { - } & \begin{array}{l}\text { Institución pública } \\
\text { (Huancavelica). }\end{array} \\
\text { - } & \begin{array}{l}\text { Institución pública } \\
\text { (Loreto). }\end{array} \\
\text { - } & \begin{array}{l}\text { Institución pública } \\
\text { (Piura). }\end{array}\end{array}$ & $\begin{array}{ll}\text { - } & \begin{array}{l}\text { ONG internacional } \\
\text { (Áncash). }\end{array} \\
\text { - } & \begin{array}{l}\text { Empresa transnacional } \\
\text { minera (Áncash). }\end{array} \\
\text { - } & \text { Empresa nacional (Ica). } \\
\text { - Empresa transnacional } \\
\text { minera (Junín). } \\
\text { - Empresa transnacional } \\
\text { petrolera (Piura). }\end{array}$ \\
\hline
\end{tabular}

Fuente: Elaboración propia.

Para los efectos del presente estudio, entiéndase por área de RR. PP. a las oficinas de comunicaciones, comunicación corporativa, comunicación institucional, imagen institucional, imagen corporativa, prensa y afines.

\subsection{Descripción de los instrumentos}

Los instrumentos se elaboraron tomando en cuenta la Escala de Likert, que consiste en un conjunto de ítems presentados en forma de afirmaciones o juicios, ante los cuales se pidió la reacción de los participantes frente a las alternativas: "totalmente de acuerdo" (TA), "de acuerdo" (DA), "ni de acuerdo ni en desacuerdo" (NAND), "en desacuerdo" (ED) y "totalmente en desacuerdo" (TD).

Los instrumentos elaborados fueron los siguientes:

- Escala de medición de la función relacional de RR. PP., de Valdez.

- Escala de medición de la función comunicacional de RR. PP., de Valdez.

- Escala de medición de la función sinérgica de RR. PP., de Valdez.

Para llegar a la versión final de cada escala se pasó por una serie de etapas. La escala original constaba en total de 145 ítems (función relacional, 34; función comunicacional, 40; función sinérgica, 41). Tras la opinión de los jueces y el estudio piloto, la versión final quedó en 73 ítems (función relacional, 29; función comunicacional, 24; y función sinérgica, 20). 
Figura 3: Jueces validadores nacionales e internacionales.

\begin{tabular}{|c|c|c|}
\hline $\begin{array}{c}\text { FUNCIÓN } \\
\text { RELACIONAL }\end{array}$ & FUNCIÓN COMUNICACIONAL & FUNCIÓN SINÉRGICA \\
\hline \multicolumn{3}{|c|}{ Jueces nacionales } \\
\hline 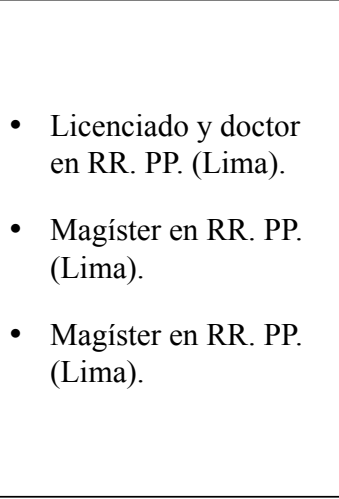 & $\begin{array}{l}\text { - } \begin{array}{l}\text { Magíster en Comunicación Social } \\
\text { (Junín). }\end{array} \\
\text { - Magíster en Comunicación Social } \\
\text { (Junín). } \\
\text { - Licenciado y funcionario estatal de } \\
\text { prensa (Lima). } \\
\text { - Licenciado en Ciencias de la } \\
\text { Comunicación y magíster en } \\
\text { Educación (Lima). } \\
\text { - Doctor en Comunicación (Piura). }\end{array}$ & 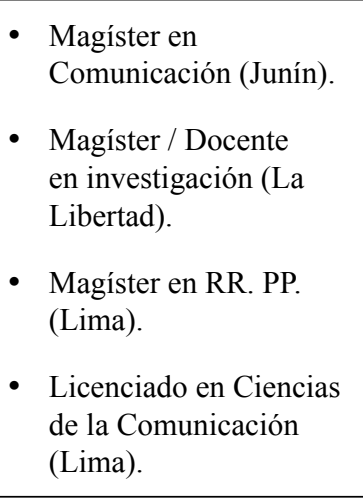 \\
\hline \multicolumn{3}{|c|}{ Jueces internacionales } \\
\hline 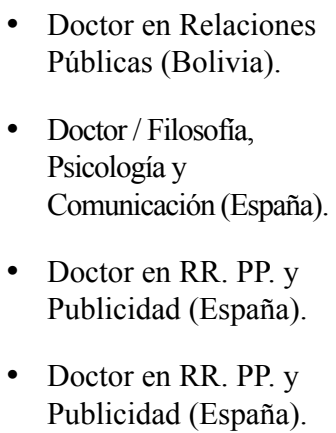 & $\begin{array}{l}\text { - Licenciado en Comunicación } \\
\text { (Argentina). } \\
\text { - Magíster en Relaciones Públicas } \\
\text { (Colombia). }\end{array}$ & $\begin{array}{l}\text { - Doctor en Comunicación } \\
\text { (Colombia). } \\
\text { - Magíster en } \\
\text { Comunicación (Ecuador) } \\
\text { - Magíster en } \\
\text { Comunicación (Chile). }\end{array}$ \\
\hline
\end{tabular}

Fuente: Elaboración propia.

\section{Marco teórico}

Luego del análisis crítico hecho tras la revisión de la literatura científica sobre las escuelas doctrinarias norteamericana, europea y latinoamericana, la explicitación de los diversos modelos teóricos y el juicio realizado sobre la práctica existente, este estudio propone la articulación desde una concepción de conjunto de un nuevo modelo teórico basado en el establecimiento de tres funciones de RR. PP. que deben estar presentes en las organizaciones públicas y privadas: la función relacional, comunicacional y sinérgica.

En esta propuesta, que se pone a consideración de la academia, se da especial énfasis al enfoque relacional, porque considera al factor humano como el elemento más importante en el complejo tejido social presente en la dinámica y el comportamiento de las organizaciones. 
Por consiguiente, se define a la función relacional de RR. PP., desde el ámbito de las ciencias sociales, como el conjunto de acciones que garantiza una coherente relación humana, fluidas relaciones de poder y una adecuada administración de la controversia pública entre los grupos de interés de una organización sea esta pública o privada.

Solórzano Hernández (2004) considera que la acción relacional está implícita en las funciones de identificar y analizar tendencias de las organizaciones y sus públicos, diagnosticar situaciones y pronosticar futuros comportamientos, asesorar a los altos niveles de la organización en la formulación de planes de relación-comunicación para realimentar la gestión estratégica. La función relacional tiene como su dimensión principal a la relación humana, que consiste en la gestión del relacionamiento para contribuir a la generación de confianza entre la organización y sus públicos. Solórzano Hernández (2000) rescata, precisamente, desde una perspectiva antropológica y cultural el valor de la relación humana como condición de legitimación de la acción comunicacional.

La generación de confianza en los públicos es el conjunto de estrategias que contribuyen a que la organización sea íntegra, competente y confiable para sus públicos. Noguero (2007: 13) advierte que las RR. PP configuran la estrategia de la confianza porque en ella reposa la credibilidad del mensaje.

Otra dimensión de la función relacional propuesta es la gestión de las relaciones de poder, que es el conjunto de acciones programadas de investigación, planificación y asesoramiento de la interacción entre la organización y los distintos grupos de interés. Porto Simões (2001) ubica a las RR. PP. como una gestión política de las organizaciones, en la que subyacen las relaciones de poder.

La tercera y última dimensión de la función relacional es el proceso de administración de la controversia pública, que es el conjunto de acciones consistente en la recreación por parte del relacionista de un escenario de intercambios polémicos entre los diversos públicos de la organización, a fin de realizar una tarea de intermediación que tiene como finalidad evitar el conflicto y las crisis que pudieran presentarse a futuro.

Mediante la administración de la controversia pública se recoge información sobre las opiniones, actitudes y comportamientos de los públicos vinculados a la organización; se escucha, dialoga y se plantean soluciones partiendo de las mismas interacciones de los públicos controvertidos. De esta manera, se apunta a la prevención del conflicto y de la crisis. Solórzano Hernández (2007) ha desarrollado en el país el concepto de la administración de la controversia pública como instrumento metodológico de las RR. PP.

De otro lado, la variable función comunicacional está basada en la comunicación como el instrumento más eficaz que tiene las RR. PP. para integrar a la organización con sus públicos, la comunidad y la sociedad. Se trata del despliegue de acciones programáticas de intercambio de mensajes y el establecimiento de roles para lograr el entendimiento mutuo organización-públicos. La función comunicacional de las RR. PP. está explícita en los cuatro modelos de RR. de Grunig y Hunt (2000). 
Una de las dimensiones de la función comunicacional es el mensaje comunicacional unidireccional sobredimensionado, que está referido a las acciones de la organización con fines de propaganda, posicionamiento de marca, encumbramiento del líder y de cobertura mediática. Otra dimensión es el mensaje comunicacional unidireccional racional, referido a las acciones prudentes y objetivas de relacionamiento entre la organización y los medios de comunicación. En este caso se respeta la decisión de los medios respecto a la información que publica sobre la organización y se colabora con ellos en el acopio de información objetiva sobre la dinámica organizacional.

Se aprecia en las organizaciones la dimensión denominada "rol bidireccional sobredimensionado", referido al conjunto de acciones programáticas de comunicación, en el que se recurre a las técnicas de las ciencias sociales para persuadir a los públicos a fin de que acepten el punto de vista de la organización y sólo sea beneficiada esta, mas no los públicos. Xifra (2003) llama a esta "función de comunicación sincrónica", porque obedece a un proceso dirigido hacia la obtención de resultados. En este caso, su finalidad es adaptar en un tiempo dado el comportamiento del público en beneficio de la organización.

Al rol bidireccional racional, dimensión que también forma parte de la función comunicacional, se le está considerando como el conjunto de acciones programáticas de comunicación en el que se recurre a las técnicas de la ciencias sociales no para persuadir sino para lograr el entendimiento mutuo organización-públicos. Xifra (2003) llama a esta "función comunicación diacrónica" cuya finalidad es negociar a lo largo del tiempo las circunstancias que beneficien tanto a la organización como a los públicos.

Otra dimensión importante de la función comunicacional es la presencia de la organización en los medios tradicionales que la circunscribimos al conjunto de acciones estratégicas de la organización conducente a lograr espacios en los medios convencionales como son la televisión, la radio, los diarios y las revistas. Xifra (2013) señala que en todos los programas de RR. PP. externas intervienen los mass media.

En la función comunicacional destaca, también, la presencia de la organización en los medios online, que está referida al conjunto de acciones estratégicas de la organización conducente a lograr la interacción organización-públicos a nivel de medios digitales, tales como el portal electrónico y cuentas oficiales en Facebook, Twitter y YouTube. Finalmente, la variable función sinérgica de las RR. PP. se da al interior de las organizaciones públicas $\mathrm{y}$ privadas $\mathrm{y}$ hace posible el cumplimiento de las otras funciones (la relacional y la comunicacional). La función sinérgica comprende los roles estratégico y gerencial de un área de RR. PP. Grunig (2006) encuadra esta función dentro de la "teoría de la excelencia" de las RR. PP.

La dimensión "rol estratégico" (perteneciente a la función sinérgica de RR. PP.) está referida a la presencia de la disciplina en el plan general de la organización, a la gestión estratégica de las funciones de RR. PP., a su participación en la alta dirección y a su ubicación en la estructura orgánica de la organización. La planificación estratégica, que es el quehacer de las RR. PP., forma parte de la función directiva (Matilla, 2007). 
La dimensión "rol gerencial" de RR. PP. es el conjunto de acciones de alto rango a cargo de un ejecutivo con formación universitaria en la especialidad, capaz de dirigir estratégicamente programas relacionales y comunicacionales en las organizaciones públicas y privadas. Grunig y Hunt (2000) aclaran que esta función debe estar separada de otras, como el marketing y el manejo de recursos humanos. Finalmente, se debe señalar que la propuesta del presente modelo teórico está basada en el constructo denominado el objeto de estudio de las RR. PP. que es el hombre de la organización desde su acción relacional y comunicacional (Pérez y Solórzano, 1999).

En la misma línea de los autores mencionados, Noguero (2007) considera que el objeto de estudio y conocimiento de las RR. PP. se circunscribe a las organizaciones y a los públicos resaltando que su metodología es propia de las ciencias sociales y que su epistemología puede significarse académicamente como en cualquier otro campo del saber. Según Bunge, la construcción de una teoría no llega a una consolidación absoluta pero unifica y profundiza las ideas en procura de dar una forma, un acabado más o menos uniforme (ápud Ayala, 2011).

El modelo teórico que se propone en la presente investigación está dentro de los alcances de lo que plantea Bunge (ibídem), es decir, se ha unificado y profundizado ideas sobre las funciones de RR. PP. que autores peruanos, latinoamericanos, europeos y norteamericanos describieron y explicaron en su oportunidad. Además, tras la unificación y profundización de ideas que se ha hecho en este estudio, se pasó al terreno empírico construyendo instrumentos de medición que han permitido medir el uso de las funciones relacional, comunicacional y sinérgica en la realidad viva de las organizaciones públicas y privadas del Perú con la validación rigurosa de prestigiosos jueces nacionales e internacionales especialistas en la materia.

\section{Análisis y discusión de resultados}

Se analizaron y discutieron los resultados sobre la base de las tres variables materia de estudio: función relacional, comunicacional y sinérgica de RR. PP. de las organizaciones públicas y privadas del Perú. En primer lugar, se analizaron y discutieron los hallazgos de las tres dimensiones de la variable función relacional de RR. PP.: subfunción relación humana, subfunción relaciones de poder y subfunción proceso de administración de la controversia pública.

En segundo lugar, se analizaron y discutieron los hallazgos de las seis dimensiones de la variable función comunicacional de RR. PP.: subfunción mensaje unidireccional sobredimensionado, subfunción mensaje unidireccional racional, subfunción rol bidireccional sobredimensionado, subfunción rol bidireccional racional, subfunción uso de medios tradicionales y subfunción uso de medios online. En tercer lugar, se analizaron y discutieron los hallazgos de las dos dimensiones de la variable función sinérgica de RR. PP.: subfunción rol estratégico de RR. PP. y subfunción rol gerencial de RR. PP. 
De acuerdo con el estudio realizado, se comprobó que las funciones de RR. PP. (relacional, comunicacional y sinérgica) están presentes en las diversas organizaciones públicas y privadas del país. Sin embargo, no existe en estas organizaciones un trabajo de articulación que permita una tarea más eficiente de RR. PP.

\subsection{Función relacional}

\subsubsection{Subfunción relación humana}

De acuerdo con los hallazgos (y al hacer la comparación entre organizaciones públicas y privadas del país), se determinó que la gestión de la relación humana que contribuye a la generación de confianza se da en un nivel alto en las organizaciones privadas de Lima; mientras que en las organizaciones privadas de provincias y públicas de Lima se registra un nivel moderado; en tanto que las organizaciones públicas de provincias registran un nivel bajo.

Esto quiere decir que en las oficinas de RR. PP. de las organizaciones privadas de Lima se planifica mejor las acciones de relacionamiento con públicos internos y públicos externos, contribuyéndose al vínculo armonioso con los diversos grupos de interés. Llama la atención el nivel bajo de relacionamiento humano en las organizaciones públicas de provincias, lo cual es cuestionable toda vez que el Estado está obligado a la entrega de un buen servicio a la ciudadanía.

Al respecto, Solórzano Hernández (2000) rescata el valor de la relación humana como condición de legitimidad de la acción comunicacional en el desarrollo de las organizaciones, sean estas públicas o privadas.

\subsubsection{Subfunción relaciones de poder}

Se encontró un nivel alto en la gestión de la subfunción relaciones de poder de las organizaciones privadas de Lima; un nivel moderado en las organizaciones privadas y públicas de provincias; y un nivel bajo en las organizaciones públicas de Lima. Se infiere que las oficinas de RR. PP. de las organizaciones privadas de Lima investigan, planifican y asesoran respecto de las relaciones de poder existente entre la organización y sus distintos grupos de interés.

Esto quiere decir que mapean a sus grupos de interés, diseñan las estrategias para amortiguar las presiones y asesoran a la alta dirección en el tema del choque organizaciónpúblicos. Las organizaciones públicas de Lima registran un nivel bajo de relaciones de poder, porque sus oficinas de RR. PP. se dedican mayormente a tareas de prensa, imagen y protocolo. Porto Simões (2001) sitúa a las RR. PP. como función política, entendida esta como la gestión de las relaciones de poder de las organizaciones respecto de sus públicos. 


\subsubsection{Subfunción proceso de administración de la controversia pública}

Se halló que las organizaciones privadas de Lima registran un nivel alto de gestión de administración de la controversia pública; mientras que las organizaciones privadas de provincias un nivel moderado; en tanto que las organizaciones públicas de Lima y de provincias registran un nivel bajo. De ello se traduce que las oficinas de RR. PP. de las organizaciones privadas de Lima recrean el escenario, es decir, promueven el intercambio de ideas controvertidas entre los públicos de la organización; son intermediarias, o sea apuestan por el diálogo; y previenen el conflicto y la crisis administrando la controversia partiendo de los puntos de vista de los grupos en contradicción.

Nuevamente se observa que las oficinas de RR. PP. de las organizaciones públicas de Lima y de provincias prácticamente no administran la controversia debido a que el rol que cumplen es mayormente mediático. Al respecto, Solórzano (2000) propone el método “tópico-dialéctico" para observar el desarrollo de las interacciones y determinar cómo son las relaciones y comunicaciones entre la organización y sus públicos. La idea es hallar en las opiniones, que fluyen de esas interacciones, la solución a las naturales controversias entre los grupos sociales.

\subsection{Función comunicacional}

\subsubsection{Subfunción mensaje unidireccional sobredimensionado}

Se determinó que la subfunción mensaje unidireccional sobredimensionado de las oficinas de RR. PP. se da en un nivel alto en las organizaciones privadas de Lima; mientras que las organizaciones privadas de provincias registran un nivel moderado; en tanto que las organizaciones públicas de Lima consignan un nivel bajo. Las organizaciones privadas de provincias registran un nivel muy bajo.

En consecuencia, las oficinas de RR. PP. de las organizaciones privadas de Lima trabajan con énfasis en buscar el impacto mediático de la organización colocando fundamentalmente a la marca en el escenario mediático. No ocurre lo mismo con las organizaciones privadas de provincias en que hay una relativa ausencia de búsqueda sobredimensionada de cobertura mediática masiva en los medios. Sobre el particular, Grunig y Hunt (2000) sostienen que se trata del modelo "agente de prensa" en que se advierte una exageración de la oficina de RR. PP. por posicionar a las organizaciones en el escenario mediático.

\subsubsection{Subfunción mensaje unidireccional racional}

Se encontró que la subfunción mensaje unidireccional racional de las oficinas de RR. PP. se da en un nivel alto en las organizaciones privadas de Lima; mientras que las organizaciones privadas de provincias registran un nivel moderado; en tanto que las organizaciones públicas de Lima consignan un nivel bajo. Finalmente, las organizaciones públicas de 
provincias registran un nivel muy bajo. Esto se traduce en que las oficinas de RR. PP. de las organizaciones privadas de Lima alcanzan información objetiva de interés público a los medios masivos de comunicación.

Las organizaciones públicas de Lima y de provincias no presentan, por lo general, a los medios de comunicación información de interés público. Sobre esto, Castillo (2004) señala que el propósito de la organización es difundir la información sin ningún atisbo de persuasión, ya que lo único que se pretende es realizar una función periodística al suministrar noticia objetiva de la organización, por lo que la información no es engañosa ni tergiversada, sino que es plenamente informativa y de interés público.

\subsubsection{Subfunción rol bidireccional sobredimensionado}

Se determinó que la subfunción rol bidireccional sobredimensionado de las oficinas de RR. PP. se da en un nivel alto en las organizaciones privadas de Lima; mientras que las organizaciones privadas de provincias registran un nivel moderado; en tanto que las organizaciones públicas de Lima y de provincias consignan un nivel muy bajo. Quiere decir que las oficinas de RR. PP. de las organizaciones privadas de Lima persuaden a sus públicos para que estos acepten el punto de vista de la organización.

Se observa que ello no ocurre con las organizaciones públicas de Lima y de provincias debido a que estas practican mayormente una comunicación unidireccional, de traslado de información a través de mensajes noticiosos hacia los públicos. Sobre el particular, Grunig y Hunt (2000) señalan que se trata del modelo "asimétrico-bidireccional" de la persuasión de masas que podía basarse en las ciencias sociales.

\subsubsection{Subfunción rol bidireccional racional}

Se halló que la subfunción rol bidireccional racional de las oficinas de RR. PP. se da en un nivel alto en las organizaciones privadas de Lima; mientras que las organizaciones privadas de provincias y públicas de Lima registran un nivel moderado; en tanto que las organizaciones públicas de provincias consignan un nivel bajo.

Se deduce, entonces, que las oficinas de RR. PP. de las organizaciones privadas de Lima investigan y planifican para tratar de lograr el entendimiento mutuo con sus públicos. Esto no se presenta en las organizaciones públicas de provincias toda vez mayormente están abocadas a una gestión unidireccional de relacionamiento con los medios. Al respecto, Castillo (2004) sostiene que en este caso, las RR. PP. tienen como función servir de mediadoras entre la organización y los públicos, buscando una comprensión mutua entre ambos, utilizando teorías de la comunicación y de las ciencias sociales, dejando de lado instrumentos de comunicación persuasiva. 


\subsubsection{Subfunción uso de medios tradicionales}

Se determinó que la subfunción uso de medios tradicionales de las oficinas de RR. PP. se da en un nivel alto en las organizaciones privadas de Lima; mientras que las organizaciones públicas de Lima y de provincias registran un nivel bajo; en tanto que las organizaciones privadas de provincias consignan un nivel muy bajo. Por consiguiente, las oficinas de RR. PP. de las organizaciones privadas de Lima recurren con mayor incidencia a la televisión, la radio, los diarios y las revistas para posicionar a la organización. Esto se entiende por la brecha digital que aún existe en el país, es decir, los mass media todavía son un filtro necesario para que las organizaciones puedan llegar a la opinión pública.

En un nivel bajo se encuentran las organizaciones públicas de Lima y de provincias; y muy bajo las privadas de provincias. Gerbner sostiene que se trata de una producción y distribución en masa de mensajes públicos y únicos que pueden llegar a grupos amplios, heterogéneos y dispersos con los mismos mensajes y en corto tiempo para crear y mantener alguna comunidad de significado y perspectiva entre tales grupos (ápud Ponce, 2001).

\subsubsection{Subfunción uso de medios online}

Se ha hallado que la subfunción uso de medios online de las oficinas de RR. PP. se da en un nivel alto en las organizaciones privadas de Lima; mientras que las organizaciones públicas de provincias registran un nivel moderado; en tanto que las organizaciones públicas de Lima y de provincias consignan un nivel bajo.

Las oficinas de RR. PP. de las organizaciones privadas de Lima recurren con mayor incidencia a los portales electrónicos, a las cuentas oficiales de Facebook, Twitter y YouTube para entablar relaciones con sus distintos públicos.

Se entiende que son estas oficinas las que administran los servicios de Internet para promover el producto, el servicio, la marca, a los líderes; escuchar, dialogar y aclarar a la opinión pública situaciones críticas de la organización. Sin embargo, registran un nivel bajo las organizaciones privadas y públicas de Lima y de provincias. Sobre esto, Sánchez (2001) señala que la Internet es en principio un eficaz vehículo de información entre las empresas y los públicos que permite el envío y la recepción de datos en tiempo real y a bajo costo.

\subsection{Función sinérgica}

\subsubsection{Subfunción rol estratégico de RR. PP.}

Se encontró que la subfunción uso del rol estratégico de las oficinas de RR. PP. se da en un nivel alto en las organizaciones privadas de Lima; mientras que se observa un nivel moderado en las organizaciones públicas y privadas de provincias y en las organizaciones públicas de Lima. De ello se deduce que en las organizaciones privadas de Lima, las 
acciones del área de RR. PP. forman parte de la gestión estratégica de la organización y que, además, investigan, planifican, asesoran y miden sus acciones relacionales y comunicacionales. Además, la oficina de RR. PP. forma parte de la alta dirección y tiene a su cargo los programas de comunicación.

Respecto a esta dimensión, Grunig (2010) precisa que una excelente función de RR. PP. se sustenta en 4 categorías que pueden ser usadas para auditar una función de RR. PP.: a) rol gerencial y no técnico; b) todo programa de comunicación debe estar inserto en el área de RR: PP.; c) practicar la comunicación con un sentido simétrico; y d) la inclusión de hombres y mujeres, sin distinción, en puestos de la alta dirección.

\subsubsection{Subfunción rol gerencial de RR. PP.}

Se encontró que la subfunción rol gerencial de las oficinas de RR. PP. se da en un nivel alto en las organizaciones privadas de Lima, mientras que se observa un nivel moderado en las organizaciones públicas de Lima. En tanto, hay un nivel bajo de las organizaciones públicas y privadas de provincias.

Ello quiere decir que las acciones de dirección del área de RR. PP. están dirigidas por personas con formación universitaria en comunicación social. En la investigación sobre RR. PP. excelentes, Grunig (2006) sostiene que se hallaron dos roles: el rol directivo (o gerente) y el rol técnico. Si el comunicador de alta dirección no tiene nivel de gerente, carecería de poder.

\section{Conclusiones}

Las principales conclusiones se organizan sobre la base de los objetivos referidos a la función relacional, comunicacional y sinérgica de RR. PP. de las organizaciones públicas y privadas tanto de Lima como de provincias.

- Se encontró que la gestión de la relación humana (que contribuye a la generación de confianza) se da en un nivel alto en las organizaciones privadas de Lima y bajo en las organizaciones públicas de provincias.

- Asimismo, se halló que la subfunción relaciones de poder registra un nivel alto en las organizaciones privadas de Lima y bajo en las organizaciones públicas de Lima y de provincias.

- Respecto a la subfunción administración de la controversia pública, se determinó un nivel alto en las organizaciones privadas de Lima y un nivel bajo en las organizaciones públicas de Lima y de provincias.

En relación con el segundo objetivo general, consistente en comparar la función comunicacional de RR. PP. de las organizaciones públicas y privadas del Perú: 
- Se encontró que la subfunción mensajes de comunicación unidireccional sobredimensionados registra un nivel alto en las organizaciones privadas de Lima, bajo en las organizaciones públicas de Lima y muy bajo en las organizaciones privadas de provincias.

- Asimismo, se determinó que las organizaciones privadas de Lima registran un nivel alto en la subfunción mensajes de comunicación unidireccional racional, bajo en las organizaciones públicas de Lima y muy bajo en las organizaciones privadas de provincias.

- Con respecto a la subfunción rol de comunicación bidireccional sobredimensionado, se halló un nivel alto en las organizaciones privadas de Lima y bajo en las organizaciones públicas de Lima y de provincias.

- En cuanto a la subfunción rol de comunicación bidireccional racional, se halló un nivel alto en las organizaciones privadas de Lima y bajo en las organizaciones públicas de provincias.

- Sobre la subfunción uso de medios tradicionales, se registró un alto nivel en las organizaciones privadas de Lima, bajo en las organizaciones públicas de Lima y de provincias y muy bajo en las organizaciones privadas de provincias.

- Finalmente, en relación a la subfunción uso de medios online, se determinó un nivel alto en las organizaciones privadas de Lima, moderado en las públicas de provincias y bajo en las organizaciones públicas de Lima y de provincias.

En relación con el tercer objetivo general, consistente en comparar la función sinérgica de Relaciones Públicas de las organizaciones públicas y privadas del Perú:

- La subfunción rol estratégico de RR. PP. registra un alto nivel en las organizaciones privadas de Lima y moderado en las organizaciones públicas y privadas de provincias.

- De otro lado, la subfunción rol gerencial de RR. PP. consigna un alto nivel a las organizaciones privadas de Lima; moderado en las organizaciones públicas de Lima y bajo en las organizaciones públicas y privadas de provincias.

\section{Fuentes consultadas}

Ayala, M. (2011). Relaciones Públicas y su concreción teórica y funcional como disciplina independiente. Análisis del ámbito latinoamericano [tesis doctoral]. Lima: Universidad de San Martín de Porres, Facultad de Ciencias de la Comunicación.

Alonso, G. y Arébalos, A. (2010).L@ revolución horizontal. El poder de la comunicación en manos de la gente. Lima, Perú: Fondo Editorial de la Universidad de San Martín de Porres. 
Arceo Vacas, J.

_(1999). Tratado de Publicidady Relaciones Públicas. Madrid: Instituto de Comunicación Institucional y Empresarial de la Universidad Complutense de Madrid.

_(1998). "Fundamento para la teoría y práctica de las Relaciones. Públicas” [CD-ROM]. Barcelona, España: Promociones y Publicaciones Universitarias.

Boiry, P. (1998). Relaciones Públicas o la estrategia de la confianza. Barcelona: Ediciones Gestión 2000.

Castillo, A. (2004). "Investigación sobre la evolución histórica de las Relaciones Públicas". Revista Historia y comunicación Social, núm. 9, pp. 43-62.

Consejo Episcopal Latinoamericano (1997). Vocabulario de términos de comunicación social. Bogotá: Litoperla Impresores TLDA.

Curtichs, J. y otros (2010). Sentido Social. La comunicación y el sentido común en la era de la Internet social. Barcelona: Editorial Profit.

Dascal, M. (2005). Controversies. Ámsterdam: John Benjamins Company.

Degregori, C. y Grompone, R. (1991). Demonios y redentores en el nuevo Perú. Una tragedia de dos vueltas. Lima: IEP.

Grunig, J.

(2010). Las Nuevas Relaciones Públicas. Lima: USMP.

_(2006). "Excelencia en la Gestión de las Relaciones Públicas y la comunicación". Ponencia presentada en el II Congreso Internacional de Relaciones Públicas. Lima.

Grunig, J. y Hunt, T. (2000). Dirección de Relaciones Públicas. Barcelona: Gestión 2000.

Matilla, C. (2007). Aportaciones para un modelo global de planificación estratégica en Relaciones Públicas y comunicación integral. Análisis de un caso: El uso de la planificación estratégica en algunas agencias y consultoras de Relaciones Públicas y Comunicación del Estado español [tesis doctoral]. Barcelona: Universidad Ramón Llull. Extraída el 1/ IX/2014 desde http://dialnet.uniroja.es/servlel/busquedadoc? $\mathrm{t}=$ relaciones + publicas \& $\mathrm{db}=3$ \&td $=$ todo

Noguero, A. (2007). "Consideraciones acerca de las Relaciones Públicas en el siglo XX y su incardinación actual en el fenómeno de la globalización”. Revista Científica de la Asociación Latinoamericana de Carreras Universitarias de Relaciones Públicas, núm. 4, pp. 4-30.

Pérez, R. y Solórzano, E. (1999). Relaciones Públicas Superiores. Una nueva pedagogía. Facultad de Ciencias de la Comunicación. Lima: USMP. 
Ponce, H. (2001). Imágenes críticas de la televisión actual. Lima: USMP.

Porto Simões, R. (2001). Relaçoes Públicas e micropolitica. San Pablo: Summus Editorial. Extraída el 25/VIII/2015 desde http://books.google.es/books?id=1W2bkvQeuKcC\&print $\mathrm{sec}=$ frontcover $\& \mathrm{dq}=$ roberto + porto + simoes $\& \mathrm{hl}=\mathrm{es} \& \mathrm{sa}=\mathrm{X} \& \mathrm{ei}=\mathrm{kyj}$ MUqSxL8idkAf9nID4 Aw\&ved $=0$ CDoQ6AEwAQ\# $\mathrm{v}=$ onepage $\& \mathrm{q}=$ roberto $\% 20$ porto $\% 20$ simoes $\& \mathrm{f}=$ false

Sánchez, A. (2001). Internet y Relaciones Públicas. Lima: USMP.

Solórzano Castro, M. (2007). Calidad de las relaciones, fluidez de la comunicación y los públicos en una Asociación Militar de Bienestar de la ciudad de Lima [tesis de maestría]. Lima: Universidad de San Martín de Porres, Facultad de Ciencias de la Comunicación, Turismo y de Psicología.

Solórzano Hernández, E.

_(2007). "Relaciones Públicas: Administrando la controversia y construyendo acuerdos. I Congreso Internacional de Relaciones Públicas". Revista Científica de la Asociación Latinoamericana de Carreras Universitarias de Relaciones Públicas, núm. 4, pp. 49-58. _(2004). "Relaciones Públicas, una respuesta a los desafíos de credibilidad de las organizaciones". Revista Cientifica de la Asociación Latinoamericana de Carreras Universitarias de Relaciones Públicas, núm. 2, pp. 35-44.

_(2000). "Relación humana, legitimidad y acción comunicacional. Propuestas teóricas y metodológicas de las Relaciones Públicas". Revista Cientifica de la Asociación Latinoamericana de Carreras Universitarias de Relaciones Públicas, núm. 1, pp. 5-11.

Xifra, J. (2003). Teorías y estructuras de las Relaciones Públicas. Madrid: McGraw-Hill. 